\title{
Synergistic Effects between Phosphorylation of Phospholamban and Troponin I Promote Relaxation at Higher Heart Rate
}

\author{
Lin Zhang, Yuan Yu, Zhen Song, Yun-Ying Wang, and Zhi-Bin Yu \\ Department of Aerospace Physiology, Fourth Military Medical University, 169\# Changlexi Road, Xi'an 710032, China
}

Correspondence should be addressed to Zhi-Bin Yu, yuzhib@fmmu.edu.cn

Received 13 February 2011; Accepted 17 June 2011

Academic Editor: Xupei Huang

Copyright ( $) 2011$ Lin Zhang et al. This is an open access article distributed under the Creative Commons Attribution License, which permits unrestricted use, distribution, and reproduction in any medium, provided the original work is properly cited.

\begin{abstract}
We hypothesized that the extent of frequency-dependent acceleration of relaxation (FDAR) would be less than that of isoproterenol-(ISO-)dependent acceleration of relaxation (IDAR) at the same increment of heart rates, and ISO may improve FDAR. Cardiac function and phosphorylation of PLB and cTnI were compared in pacing, ISO treatment, and combined pacing and ISO treatment in isolated working heart. The increase in cardiac output and the degree of relaxation was less in pacing than in ISO treatment at the same increment of heart rates. The increasing stimulation frequency induced more significant relaxant effect in ISO perfusion than that in physiological salt perfusion. The pacing only phosphorylated PLB at Thr17, but ISO induced phosphorylation of cTnI and PLB at Ser16 and Thr17. Those results suggest that the synergistic effects of PLB and cTnI induce higher degree of relaxation which makes a sufficient diastolic filling of the ventricle at higher heart rate.
\end{abstract}

\section{Introduction}

Heart rate is a fundamental physiological modulator of cardiac function in all mammalian hearts. Regardless of whether the force-frequency relationship is positive or negative, an increase in heart rate is always associated with an acceleration of relaxation, named as frequency-dependent acceleration of relaxation (FDAR) [1]. This FDAR ensures the proper relaxation and diastolic filling of the ventricle at higher heart rates. Previous studies indicate that $\mathrm{Ca}^{2+} /$ Calmodulin-dependent kinase II (CaMKII) is a prime candidate in sensing and mediating frequency-dependent effects [2]. The CaMKII has at least four substrates involved in excitation-contraction coupling in the heart [3]. Published studies demonstrate that CaMKII can phosphorylate ryanodine receptor (RyR) [4], sarcoplasmic reticulum $\mathrm{Ca}^{2+}$-ATPase (SERCA) [5], phospholamban (PLB) [6], and the L-type $\mathrm{Ca}^{2+}$ channels (LTCC) [7]. The phosphorylation of LTCC and RyR is related to modulation to systolic function. It has been reported that LTCC activation by CaMKII is involved in the development of early afterdepolarizations and arrhythmias in vitro and in vivo [8]. Huke and Bers found that FDAR did not rely on phosphorylation of RyR [1]. Valverde et al. reported that SERCA phosphorylation by CaMKII was not involved in FDAR [9]. Thus, a very likely target of FDAR is PLB, an inhibitor of SERCA [10]. When PLB becomes phosphorylated at Thr-17 by CaMKII, the inhibition is relieved, and $\mathrm{Ca}^{2+}$ uptake of SR is accelerated [10]. However, increasing stimulation frequency still induces FDAR in PLB knockout mouse hearts [11]. Therefore, the molecular mechanism of FDAR is still controversial.

Activation of the sympathetic nervous system leads to the secretion of catecholamines, which stimulate $\beta$-adrenergic receptors in the heart. Isoproterenol-(ISO-)dependent acceleration of relaxation (IDAR) is another important intrinsic regulator to cardiac function. Stimulation of $\beta$-adrenergic receptor ( $\beta$-AR) by ISO activates Gs, which in turn activates adenylate cyclase and increases the formation of cAMP within cardiomyocytes. Elevated levels of cAMP increases the activation of protein kinase A (PKA) which phosphorylates intracellular targets such as LTCC, RyR, PLB, cardiac troponin I (cTnI), and myosin binding protein $\mathrm{C}$ (MyBPC). Phosphorylation of LTCCs enhances $\mathrm{Ca}^{2+}$ influx which increases shortening amplitude or force of contraction 
[7]. Recently, MacDonnell et al. reported that adrenergic regulation of cardiac contractility did not involve phosphorylation of the cardiac RyR at serine-2808 residue [12]. Phosphorylation of MyBPC does not appear to have any effect on myofibrillar $\mathrm{Ca}^{2+}$ sensitivity which modulates relaxation but accelerated the kinetics of force development [13]. Phosphorylated MyBPC may link to myocardial protection in ischemia $[14,15]$. The elevated intracellular $\mathrm{Ca}^{2+}$ concentration increases the activity of SERCA which indirectly accelerates relaxation of cardiomyocytes. The phosphorylated PLB at Ser-16 and Thr-17 by PKA also enhanced the activity of SERCA. The phosphorylation of cTnI reduced the $\mathrm{Ca}^{2+}$ sensitivity of myofibrils which facilitates $\mathrm{Ca}^{2+}$ dissociation from myofibrils [16]. Therefore, phosphorylation of PLB and cTnI may be two synergistic modulators to accelerate relaxation in ISO stimulation.

The increasing frequency of stimulation only phosphorylates one site of PLB, but not cTnI. Due to lack of the synergistic effect between PLB and cTnI, we hypothesized that the extent of FDAR would be less than that of IDAR at the same increment of heart rate, the less extent of FDAR may have a limitation on the sufficient diastolic filling of the ventricle with increasing frequency of stimulation, and ISO may improve FDAR. Therefore, the aim of the present study was to compare the cardiac function and extent of relaxation between FDAR and IDAR at the same increment of heart rate and further to observe the extent of FDAR during ISO stimulation.

\section{Materials and Methods}

2.1. Preparation of Isolated Working Heart. Sprague-Dawley rats weighing $220 \pm 10 \mathrm{~g}$ were used. The animal procedure described here has been approved by the Animal Care and Use Committee at the Fourth Military Medical University.

The isolated crystalloid-perfused rat heart has previously been described [17]. Rats were heparinized (500 U i.p.) and anesthetized (sodium pentobarbital, $40 \mathrm{mg} / \mathrm{kg}$ i.p.). Hearts were excised, immediately mounted, and perfused on a Langendorff apparatus (Radnoti, USA) at a constant pressure of $60 \mathrm{mmHg}$ with Krebs-Henseleit solution (in mM: $118 \mathrm{NaCl}$, $4.7 \mathrm{KCl}, 2.25 \mathrm{MgSO}_{4}, 2.25 \mathrm{CaCl}_{2}, 23.8 \mathrm{NaCO}_{3}, 1.2 \mathrm{NaH}_{2} \mathrm{PO}_{4}$, $0.32 \mathrm{EDTA}$, and $11.5 \mathrm{D}$-glucose) in a nonrecirculating retrograde mode. The time from excision to perfusion was less than 60 seconds. The perfusate was oxygenated with a gas mixture of $95 \% \mathrm{O}_{2}-5 \% \mathrm{CO}_{2}$, achieving $\mathrm{pH}$ of 7.39 to 7.41 . Left atrium was cannulated through the pulmonary vein with steel cannula (inside diameter: $1.8 \mathrm{~mm}$; outside diameter: $2.0 \mathrm{~mm}$ ). In order to detect the intraventricular pressure, an ultraminiature pressure catheter transducer (model SPR671; Millar Instruments, Houston, USA) was put into the left ventricle through the left atrium. The preload was set up at $10 \mathrm{mmHg}$. The heart was then switched from the Langendorff to a working mode. The aortic and coronary flow was recorded at the 1st, 5th, 10th, and 15th min at the working heart mode. Electrodes placed on the aortic cannula and the apex of the ventricle were connected to a square wave stimulator (Aurora Scientific Inc., Canada, $5 \mathrm{~ms}$ pulse duration, $20 \mathrm{~V}$ pulse amplitude). Hearts were paced at
300,360 , and 420 beats/min in the absence and the presence of isoproterenol (ISO, Sigma Chemical Co.). The hearts were superfused with $1.0,10.0$, and $20.0 \mathrm{nM}$ of ISO for at least $5 \mathrm{~min}$. The aortic flow, coronary flow, heart rate, left ventricular end-systolic (LVESP) and end-diastolic (LVEDP) pressure, and maximal rates of left ventricular pressure development $\left(+\mathrm{dP} / \mathrm{dt}_{\max }\right)$ and relaxation $\left(-\mathrm{dP} / \mathrm{dt}_{\max }\right)$ were measured to evaluate the contractile function of isolated working heart. Cardiac output is equal to aortic flow plus coronary flow. Data was acquired and analyzed using a PowerLab system and Chart software (ADInstruments Inc., Sydney, Australia).

\subsection{Intracellular $\mathrm{Ca}^{2+}$ Measurement of the Single Cardiomy-} ocyte. The ventricular myocyte isolation was the same as that described previously [18]. The cannulated hearts were mounted on a Langendorff perfusion apparatus and perfused with $\mathrm{Ca}^{2+}$-free Joklik's modified minimum essential medium (Sigma-Aldrich, St. Louis, Mo, USA) containing $10 \mathrm{mM}$ HEPES, $0.1 \%$ bovine serum albumin (BSA). After $5 \mathrm{~min}$, the perfusate was switched to a circulating enzyme solution containing $0.08 \%$ collagenase I (Sigma-Aldrich) for $30 \mathrm{~min}$. All the perfusion procedure was performed at $37^{\circ} \mathrm{C}$ in a constant flow, and the perfusion pressure was monitored. Then the ventricular tissues were chopped, and the cardiomyocytes were dispersed gently by a wide tipped pipette. The cell suspension was filtered through $200-\mu$ m nylon mesh. After $30 \mathrm{~min}$, the cells were resuspended in Joklik's medium containing $1 \% \mathrm{BSA}$ and slowly recovered $\mathrm{Ca}^{2+}$ concentration to $1.25 \mathrm{mM}$.

The intracellular $\mathrm{Ca}^{2+}$ concentration of cardiomyocytes was measured within 6 hours after isolation. Ventricular myocytes were loaded with fura- $2 \mathrm{AM}(5 \mu \mathrm{M})$ for $30 \mathrm{~min}$ in the dark at room temperature. Cells were then transferred into a $0.2 \mathrm{~mL}$ chamber which was on the stage of an inverted microscope (OlympusX71, Tokyo, Japan). Cells were superfused with Tyrode solution (in mM: $132 \mathrm{NaCl}, 4.8 \mathrm{KCl}, 1.2$ $\mathrm{MgCl}_{2}, 1.8 \mathrm{CaCl}_{2}, 5.0$ sodium pyruvate, $10 \mathrm{HEPES}$, and 10 D-glucose, $\mathrm{pH} 7.40$ ) at a flow rate of $0.2 \mathrm{~mL} / \mathrm{min}$ at $37^{\circ} \mathrm{C}$. Electric field stimulus (rectangle wave, $15 \mathrm{~V}, 5 \mathrm{~ms}, 1.0 \mathrm{~Hz}$ ) was given by the stimulator. Then the stimulation frequency increased to $2 \mathrm{~Hz}, 5 \mathrm{~Hz}$, and $10 \mathrm{~Hz}$ with or without ISO perfusion $(1,10$, and $20 \mathrm{nM})$.

Intracellular $\mathrm{Ca}^{2+}$ concentration was measured by whole cell photometry (DeltaRam, Photon Technology International, USA). The emission ratio at $510 \mathrm{~nm}$, during alternate excitation at $340 \mathrm{~nm}$ and $380 \mathrm{~nm}$ was used to determine intracellular $\mathrm{Ca}^{2+}$ concentration. Fluorescence emission was measured at 240 points/s for each of the excitation wavelengths. Background fluorescence values were determined at each excitation wavelength. These background values were subtracted from the recordings made at each wavelength during each experiment. Data were acquired and analyzed by Felix software (Version 1.4, Photon Technology International). $\mathrm{Ca}^{2+}$ transient amplitudes were the difference between systolic and diastolic $\mathrm{Ca}^{2+}$.

2.3. Western Blotting. The expressions of cTnI, total and Ser16-phosphorylated and Thr-17-phosphorylated PLB were 


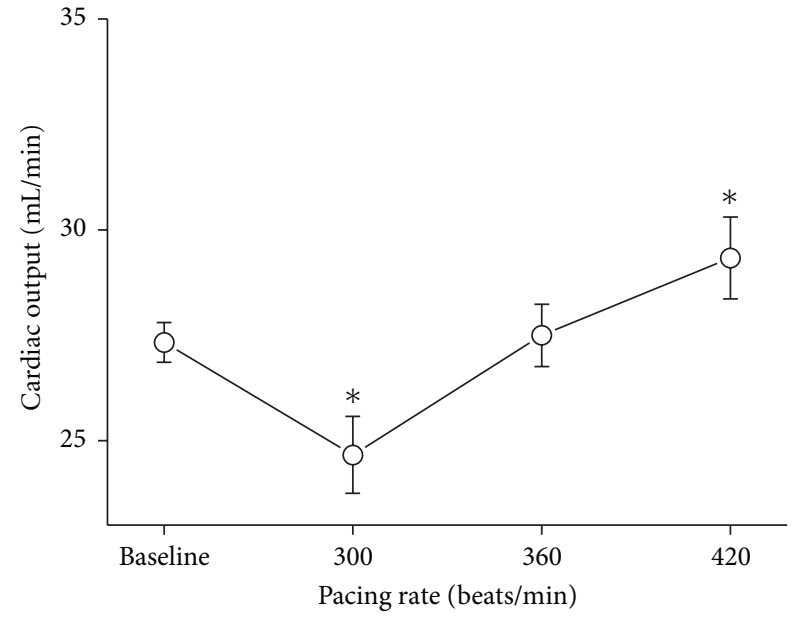

(a)

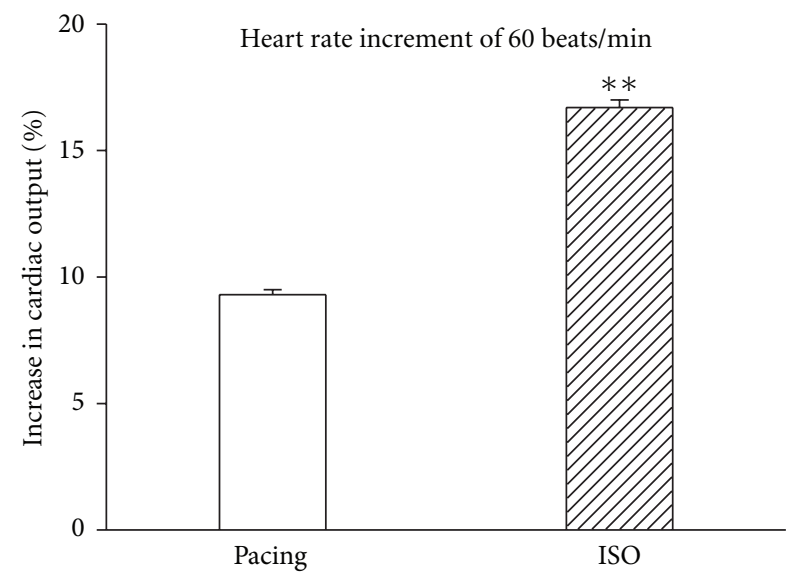

(c)

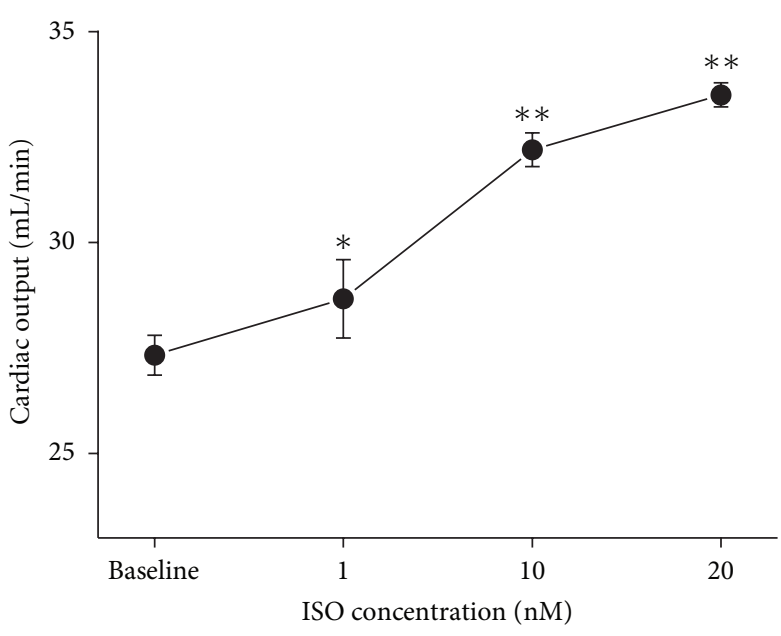

(b)

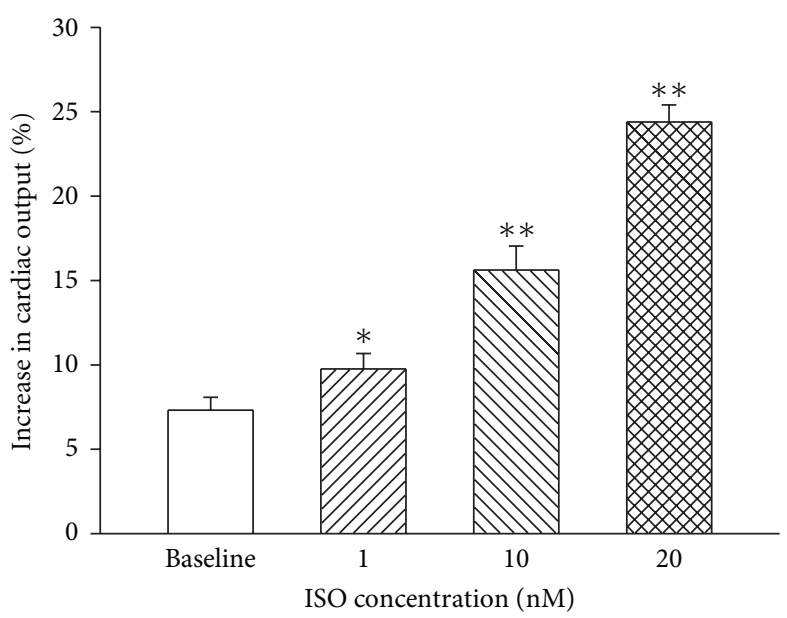

(d)

FIGURE 1: Cardiac output of isolated working hearts in the pacing and ISO treatment. All data are mean \pm SEM. (a) Cardiac output of isolated working hearts in different rates of pacing. The $n=6$ hearts. ${ }^{*} P<0.05$ versus baseline. (b) Cardiac output in different concentrations of ISO treatment. The $n=6$ hearts in each ISO concentration. ${ }^{*} P<0.05$ or ${ }^{* *} P<0.01$ versus baseline. (c) The percentage increases in cardiac output in the heart rate increment of 60 beats/min. The $n=6$ hearts for each group. ${ }^{*} P<0.05$ versus the pacing group. (d) The percentage increases in cardiac output at the pacing of 420 beats/min without and with ISO treatment. The $n=6$ hearts in each ISO concentration. ${ }^{*} P<0.05$ or ${ }^{*} P<0.01$ versus baseline.

detected by Western blotting. Left ventricular myocardium was homogenized in a buffer containing $50 \mathrm{mM}$ potassium phosphate buffer ( $\mathrm{pH} 7.0$ ) $0.5 \mathrm{mM}$ DTT, $1 \mathrm{mM}$ EDTA, $0.3 \mathrm{mM}$ PMSF, and phosphatase inhibitor cocktail $(1: 100$, Sigma-Aldrich). Samples were subjected to SDS-PAGE in polyacrylamide gels $(12 \%$ or $14 \%$ depending on protein molecular weight). After electrophoresis, protein was electrically transferred to nitrocellulose membrane $(0.45 \mu \mathrm{m}$ pore size) using a Bio-Rad semidry transfer apparatus. The blotted nitrocellulose membranes were blocked with $1 \%$ bovine serum albumin in Tris-buffered saline $(150 \mathrm{mM}$ $\mathrm{NaCl}, 50 \mathrm{mM}$ Tris- $\mathrm{HCl}$, and $\mathrm{pH}$ 7.5) and incubated with mouse monoclonal anti-TnI (TnI-1, 1:4,000; Courtesy of Dr. J.-P. Jin), mouse monoclonal anti-PLB (1:1000; Cell Signaling Technology, Inc. (CST), Danvers, Mass, USA), rabbit polyclonal anti-Ser-16-phosphorylated phospholamban
(Phospho16-PLB, $1: 1000$; CST) and rabbit polyclonal antiThr-17-phosphorylated phospholamban (Phospho17-PLB, 1:1000; Santa Cruz Biotechnology, Inc., Santa Cruz, Calif, USA) in TBS containing $0.1 \%$ BSA at $4^{\circ} \mathrm{C}$ overnight. The membranes were visualized with IRDye $680 \mathrm{CW}$ goat antimouse or IRDye $800 \mathrm{CW}$ goat antirabbit secondary antibodies $(1: 10,000$, for $90 \mathrm{~min}$ at room temperature) using an Odyssey scanner (LI-COR Biosciences, Lincoln, Neb, USA). Quantification analysis of blots was performed with the Scion Image software.

2.4. Statistical Analysis. Data are expressed as mean \pm SEM. Differences between groups were evaluated using one-way analysis of variance (ANOVA). When ANOVA indicated significance, a Dunnett post-hoc test was performed. $P<0.05$ was considered statistically significant. 


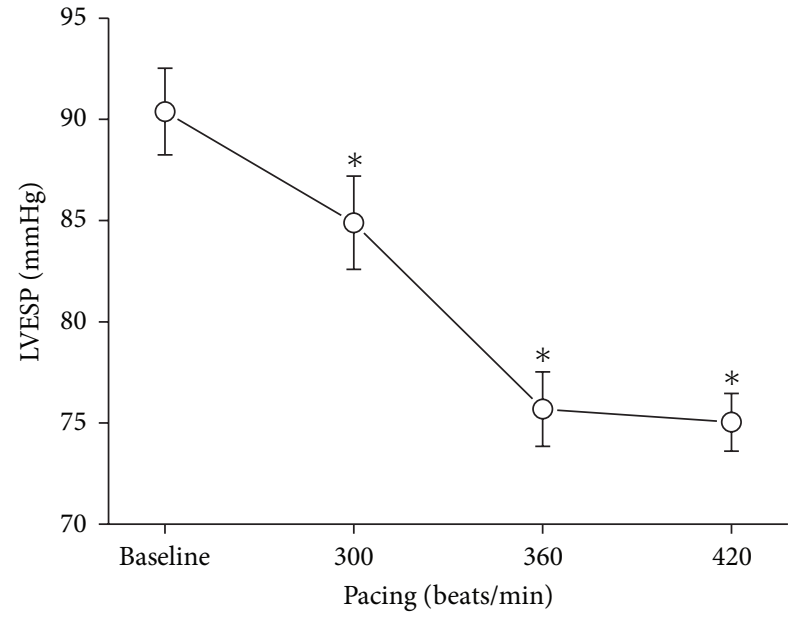

(a)

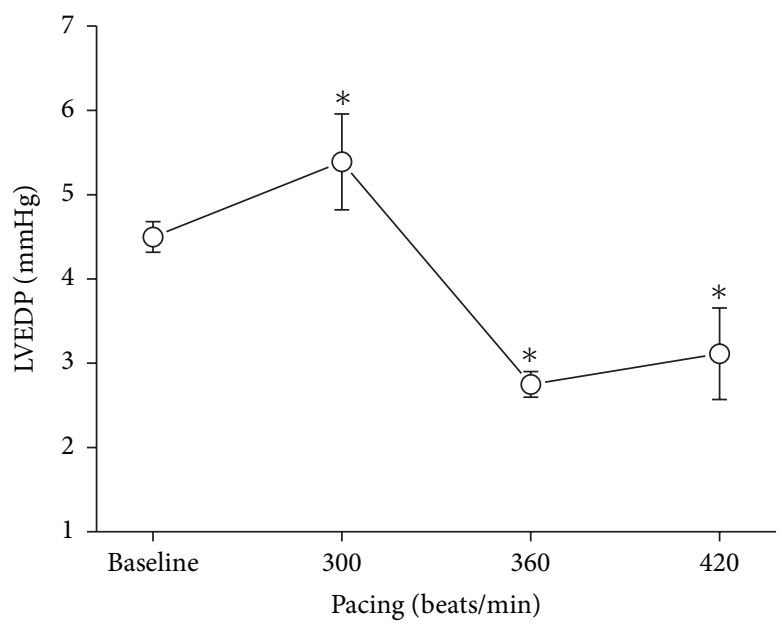

(c)

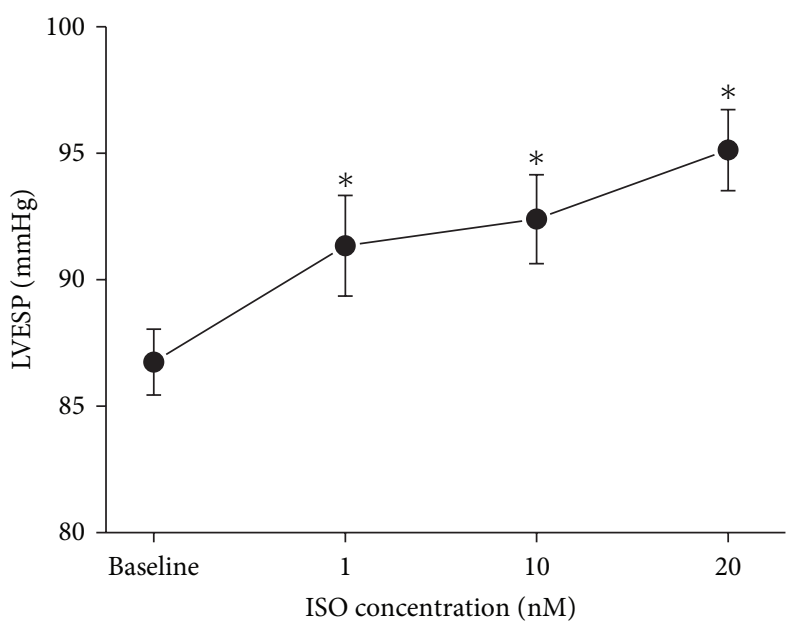

(b)

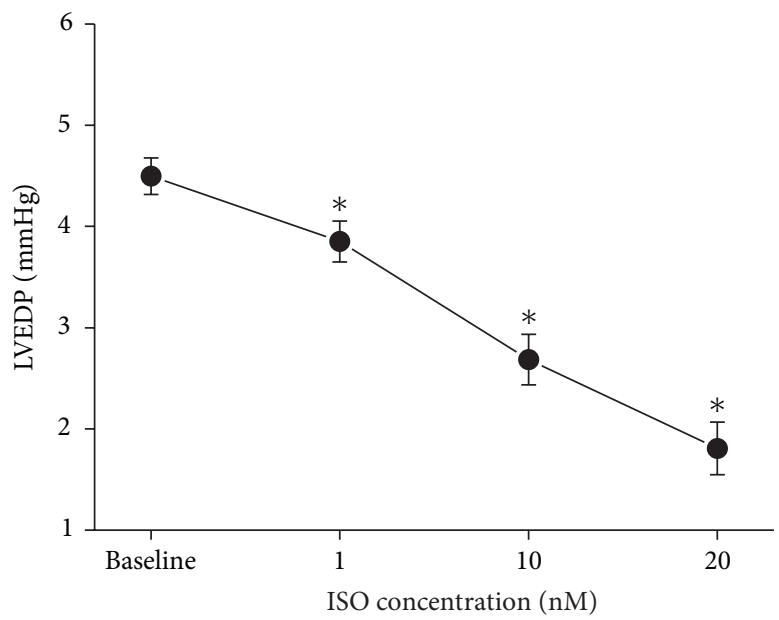

(d)

FIGURE 2: Left ventricular end-systolic pressure (LVESP) and end-diastolic pressure (LVEDP) of working hearts in the pacing and ISO treatment. (a) and (c) LVESP and LVEDP during the pacing. (b) and (d) LVESP and LVEDP in ISO treatment. Data are mean \pm SEM, $n=6$ hearts for each ISO concentration in each group. ${ }^{*} P<0.05$ versus baseline.

\section{Results}

3.1. Systolic and Diastolic Function of Isolated Working Hearts during the Pacing and ISO Treatment. At the preload of $10 \mathrm{mmHg}$ and afterload of $60 \mathrm{mmHg}$, the intrinsic heart rate of working heart was $236.4 \pm 6.4$ beats/min in the baseline group. Heart rates increased to $259.9 \pm 7.3,288.1 \pm 4.1$, and $316.7 \pm 6.3 \mathrm{beat} / \mathrm{min}$ in 1,10 , and $20 \mathrm{nM}$ ISO stimulation, respectively. At baseline, cardiac output was $27.3 \pm$ $0.5 \mathrm{~mL} / \mathrm{min}$. Cardiac output decreased to $24.7 \pm 0.9 \mathrm{~mL} / \mathrm{min}$ at the pacing of 300 beats $/ \mathrm{min}$, then increased to $27.5 \pm$ $0.7 \mathrm{~mL} / \mathrm{min}$ and $29.3 \pm 0.9 \mathrm{~mL} / \mathrm{min}$ at the pacing of 360 and 420 beats/min (Figure 1(a)). Cardiac output increased to $28.7 \pm 0.9,32.2 \pm 0.4$, and $33.5 \pm 0.3 \mathrm{~mL} / \mathrm{min}$ in 1 , 10 , and $20 \mathrm{nM}$ ISO treatment, respectively, (Figure 1(b)). While the increment of heart rate was similar at 60 beats $/ \mathrm{min}$, the percentage increase in cardiac output was higher in ISO-treated group than in the pacing group (Figure 1(c)). Cardiac output had about $7 \%, 10 \%, 15 \%$, and $24 \%$ increases, respectively, at the pacing of 420 beats/min without and with 1,10 , and $20 \mathrm{nM}$ ISO treatment compared to the baseline at the intrinsic heart rate (Figure 1(d)).

LVESP decreased with the increasing pacing rate (Figure 2(a)). In the opposite, LVESP increased with the increasing concentrations of ISO at the intrinsic heart rate (Figure 2(b)). LVEDP showed a significant increase at 300 beats/min but decreased progressively at 360 and 420 beats/min (Figure 2(c)). LVEDP decreased progressively with the increasing concentrations of ISO at the intrinsic heart rate (Figure $2(\mathrm{~d})$ ).

The $\pm \mathrm{dP} / \mathrm{dt}_{\max }$ decreased significantly at the pacing of 300 beats/min and increased at the pacing of 360 and 420 beats/min (Figure $3(\mathrm{a})$ ). In contrast, $\pm \mathrm{dP} / \mathrm{dt}_{\max }$ elevated progressively with the increasing concentrations of ISO (Figure $3(\mathrm{~b})$ ). $\mathrm{TR}_{75}$, time from peak to $75 \%$ relaxation of left ventricular pressure, is another index of cardiac diastolic function. Both the pacing and ISO stimulation reduced $\mathrm{TR}_{75}$ (Figures 3(c) and 3(d)). 


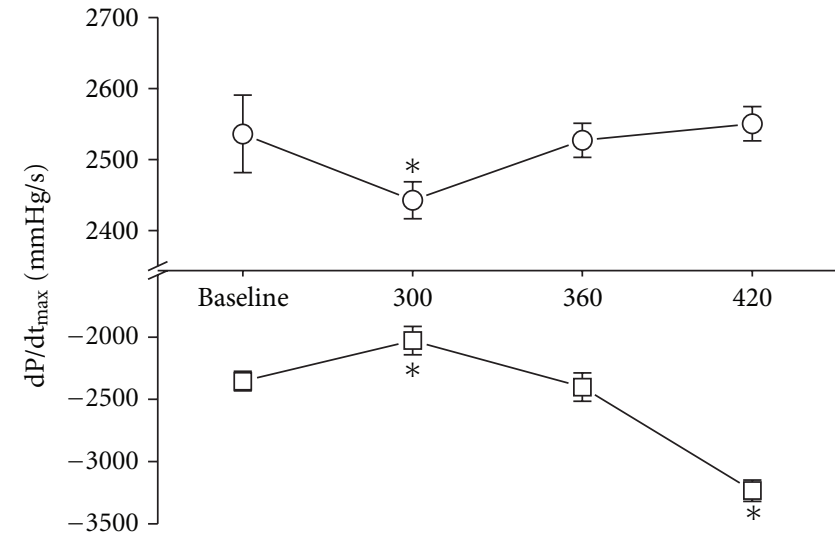

Pacing (beats/min)

(a)

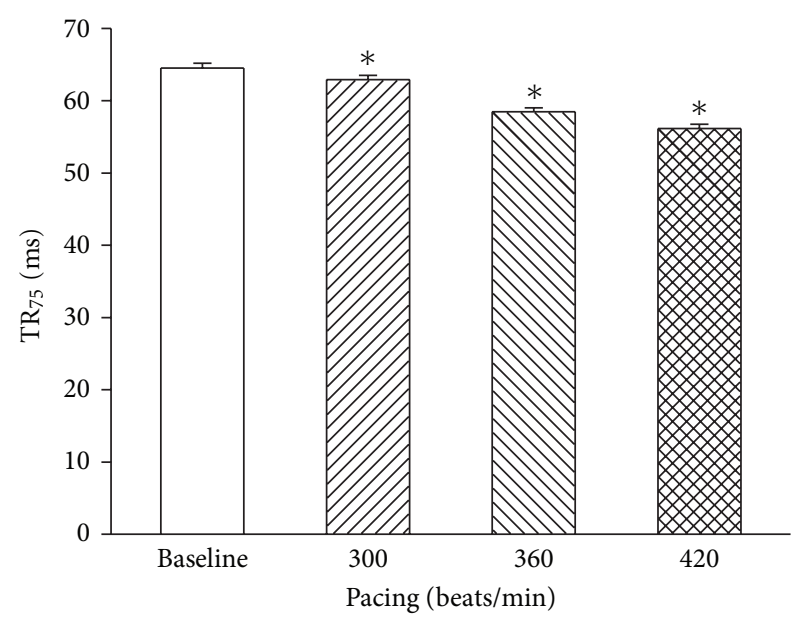

(c)

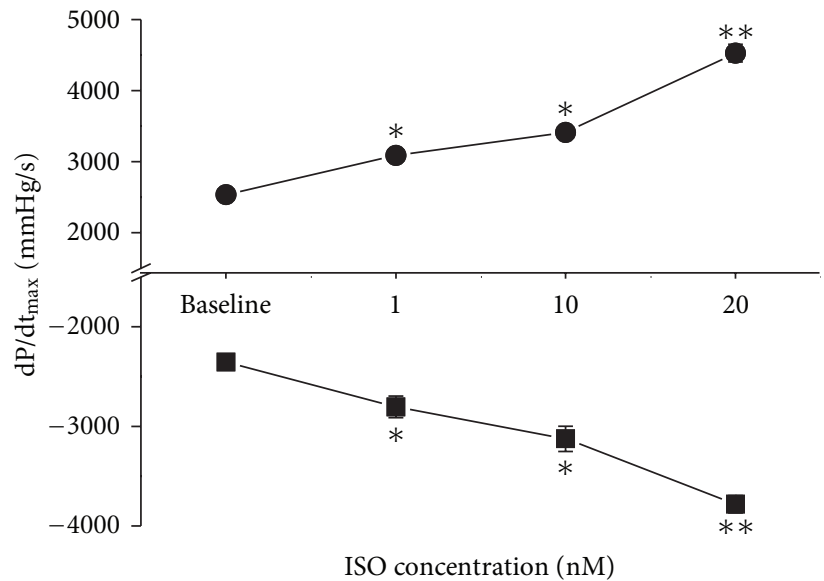

(b)

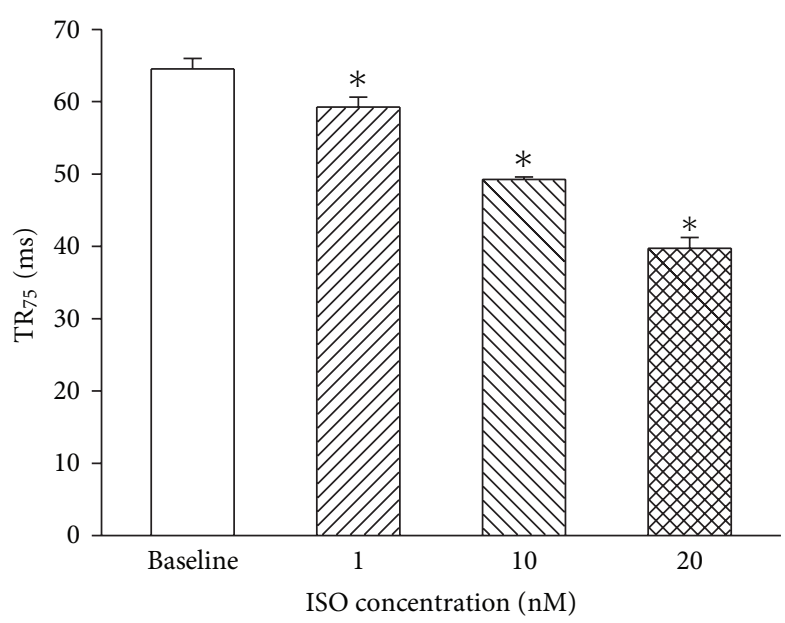

(d)

FIGURE 3: Maximal rates of left ventricular pressure development and relaxation $\left( \pm \mathrm{dP} / \mathrm{dt}_{\max }\right)$ and time from peak to $75 \%$ relaxation $\left(\mathrm{TR}_{75}\right)$ of left ventricular pressure. (a) and (c) $\pm \mathrm{dP} / \mathrm{dt}_{\max }$ and $\mathrm{TR}_{75}$ under different rates of pacing. (b) and (d) $\pm \mathrm{dP} / \mathrm{dt}_{\max }$ and $\mathrm{TR}$ 75 in different concentrations of ISO treatment. Data are mean \pm SEM, $n=6$ hearts for each ISO concentration in each group. ${ }^{*} P<0.05$ or ${ }^{* *} P<$ 0.01 versus baseline.

The percentage increases in $\pm \mathrm{dP} / \mathrm{dt}_{\max }$ between the pacing of 420 beats/min and intrinsic heart rate groups without ISO stimulation were $7.1 \pm 1.0 \%$ and $9.7 \pm 1.1 \%$, respectively, (the baseline values in Figure 4(a)). Under ISO stimulation, the pacing of 420 beats/min induced an additional enhancement. The percentage increases in $\pm \mathrm{dP} / \mathrm{dt}_{\max }$ between the pacing of 420 beats $/ \mathrm{min}$ and intrinsic heart rate groups increased progressively with the increasing concentrations of ISO (Figure $4(\mathrm{a})$ ). The pacing of 420 beats $/ \mathrm{min}$ led about $13 \%$ shortening in $\mathrm{TR}_{75}$ without ISO stimulation. Under ISO treatment, the pacing of 420 beats/min showed stronger relaxant effects than that of the baseline group (Figure 4(b)).

3.2. Intracellular $\mathrm{Ca}^{2+}$ Concentrations of the Cardiomyocyte in the Pacing and ISO Treatment. The resting intracellular calcium concentration $\left(\left[\mathrm{Ca}^{2+}\right]_{i}\right)$ increased with the increasing frequency of stimulation; higher frequency of stimulation (i.e., $>5 \mathrm{~Hz}$ ) induced a significant elevation in resting $\left[\mathrm{Ca}^{2+}\right]_{i}$ of cardiomyocytes (Figure 5(a)). The peak $\left[\mathrm{Ca}^{2+}\right]_{i}$ increased to a maximal level at $5 \mathrm{~Hz}$, then decreased slightly at $10 \mathrm{~Hz}$. In contrast, the resting $\left[\mathrm{Ca}^{2+}\right]_{i}$ decreased slightly, but the peak $\left[\mathrm{Ca}^{2+}\right]_{i}$ kept elevating with the increasing concentrations of ISO (Figure 5(b)). Under ISO perfusion, the increasing degrees of resting $\left[\mathrm{Ca}^{2+}\right]_{i}$ were less compared with non ISOtreated group while the frequency of stimulation increased from 300 to 420 beats/min (Figure 5(a)). The peak $\left[\mathrm{Ca}^{2+}\right]_{i}$ still elevated with the increasing frequency of stimulation during different concentrations of ISO treatment. The peak $\left[\mathrm{Ca}^{2+}\right]_{i}$ reached the maximal level at $10 \mathrm{~Hz}$ under $20 \mathrm{nM}$ ISO treatment (Figure 5(a)).

$\mathrm{TD}_{75}$, the time from peak to $75 \%$ decay in calcium transient, reduced both under increasing frequency of stimulation and ISO perfusion. The degrees of reduction in $\mathrm{TD}_{75}$ were lower in stimulation group than in ISO-treated group (Figures 5(c) and 5(d)). and Thr17 in the Pacing and ISO Treatment. Figure 6(a) 


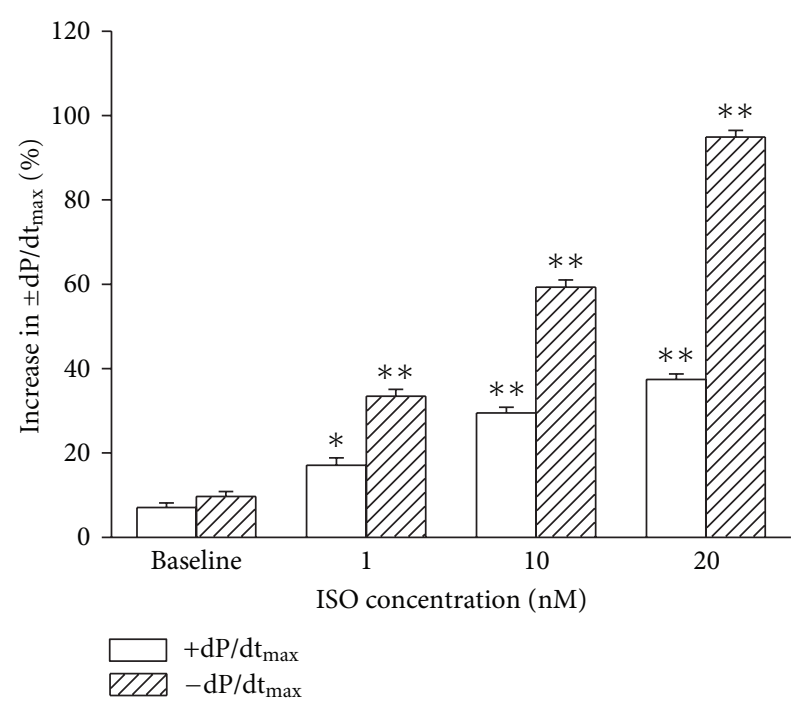

(a)

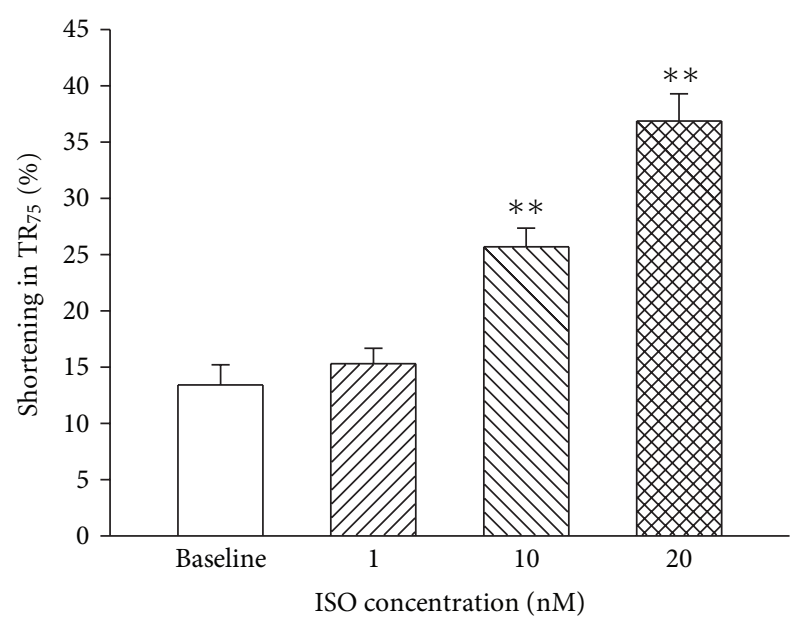

(b)

FIgURE 4: Percentage increases in $\pm \mathrm{dP} / \mathrm{dt}_{\max }$ and $\mathrm{TR}_{75}$ under combined effect of the pacing and ISO. (a) The percentage increases in $\pm \mathrm{dP} / \mathrm{dt}_{\max }$ between the pacing of 420 beats/min and intrinsic heart rate without and with ISO treatment. (b) The percentage shortenings in $\mathrm{TR}_{75}$ between the pacing of 420 beats/min and intrinsic heart rate without and with ISO treatment. Data are mean \pm SEM, $n=6$ hearts for each ISO concentration in each group. ${ }^{*} P<0.05$ or ${ }^{* *} P<0.01$ versus baseline.

shows the representative Western blots of cTnI and phosphorylated cTnI at Ser23/24. There were no differences in expression of $\mathrm{cTnI}$ in the pacing, ISO treatment, and pacing plus ISO treatment groups (Figure 6(b)). Phosphorylation of cTnI at Ser23/24 showed a dose-dependent increase in ISO perfusion. But the pacing had no effect on phosphorylation of cTnI at Ser23/24 in basic and ISO-treated condition (Figure 6(c)).

Figure 7(a) shows the representative Western blots of PLB, phosphorylated PLB at Ser16, and Thr17 in isolated working hearts under the pacing and different concentrations of ISO perfusion. Total PLB, which is the sum of pentameric and monomeric PLB, did not change under the pacing and ISO perfusion (Figure 7(b)). The phosphorylation of PLB at Ser16 and Thr17 also showed a dosedependent increase in ISO stimulation. The degrees of phosphorylated PLB at Ser16 elevated from $39.3 \pm 1.4 \%$ in baseline to $50.2 \pm 1.7 \%$ in $20 \mathrm{nM}$ ISO treatment, and the degrees of phosphorylated PLB at Thr17 increased from $13.9 \pm 0.6 \%$ in baseline to $22.5 \pm 0.6 \%$ in $20 \mathrm{nM}$ ISO treatments (Figures $7(\mathrm{c})$ and $7(\mathrm{~d})$ ). The phosphorylation of PLB at Thr17 increased with the increasing pacing rate, but the pacing did not alter the phosphorylation of PLB at Ser16. During $10 \mathrm{nM}$ and $20 \mathrm{nM}$ ISO perfusion, the pacing induced an additional increase in the phosphorylation of PLB at Ser16 in a frequency-dependent manner (Figure 7(d)).

\section{Discussion}

4.1. Coordinated Activation of $\mathrm{Ca}^{2+}$ Handing Proteins in Excitation-Contraction Coupling Is Important to Considerably Accelerate Relaxation in ISO Stimulation. In the present study, the increase in maximal rate of relaxation and the shortening in $\mathrm{TR}_{75}$ were lower in the pacing than in ISO stimulation (Figure 4(a)). It indicates that the extent of FDAR is less than that of IDAR. FDAR is an important intrinsic regulator to cardiac function in physiological condition. However, the mechanism of FDAR is still controversial. Some studies reported that FDAR may be related to the activation of CaMKII, phosphorylation of PLB, and the increase of SERCA activity. There are evidences to indicate that inhibition of CaMKII by KN-93 [9], CaMKII and PLB knock-out [11, 19], or inhibition of SERCA also shows an acceleration of relaxation in the increasing stimulation frequency [11]. We observed that the pacing only increased the phosphorylation of PLB at Thr-17 site (Figure 7). The increase in Thr-17 phosphorylation of PLB was associated with FDAR, but the relaxation effect was weak. The Thr-17 phosphorylation of PLB can relieve the inhibition to SERCA. Since the activation of SERCA by Thr- 17 phosphorylation of PLB is low, the resting intracellular $\mathrm{Ca}^{2+}$ concentration slightly elevated with an increasing stimulation frequency (Figure 5(a)). In contrast, ISO treatment showed not only the phosphorylation of PLB at Ser-16 and Thr-17 sites, but also the cTnI phosphorylation (Figure 7(a)). Several researches have indicated that PLB phosphorylation by ISO stimulation is a dominant mediator to the acceleration of relaxation in PLB knock-out mouse [20]. But it should not be ignored that the effect of cTnI phosphorylation was induced by ISO treatment on the acceleration of relaxation.

Early investigations indicate that phosphorylation of cTnI may not be important in the relaxant effect of ISO stimulation. Johns et al. found no effect of cTnI phosphorylation by PKA on the relaxation in skinned guinea pig ventricular trabeculae [21], and the precise experimental conditions may be important in this regard. Recently, many studies 


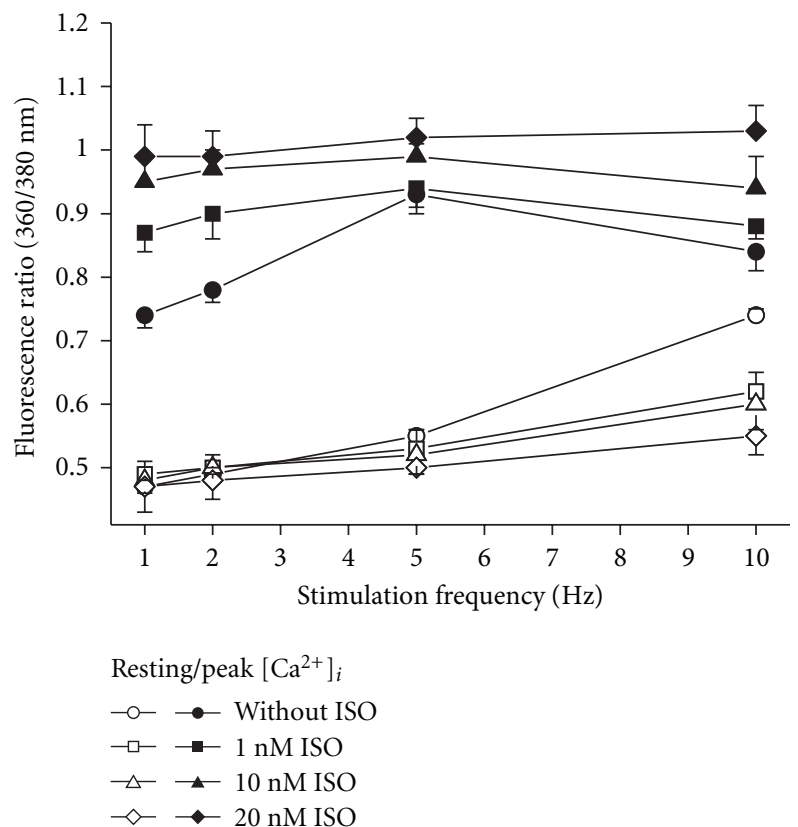

(a)

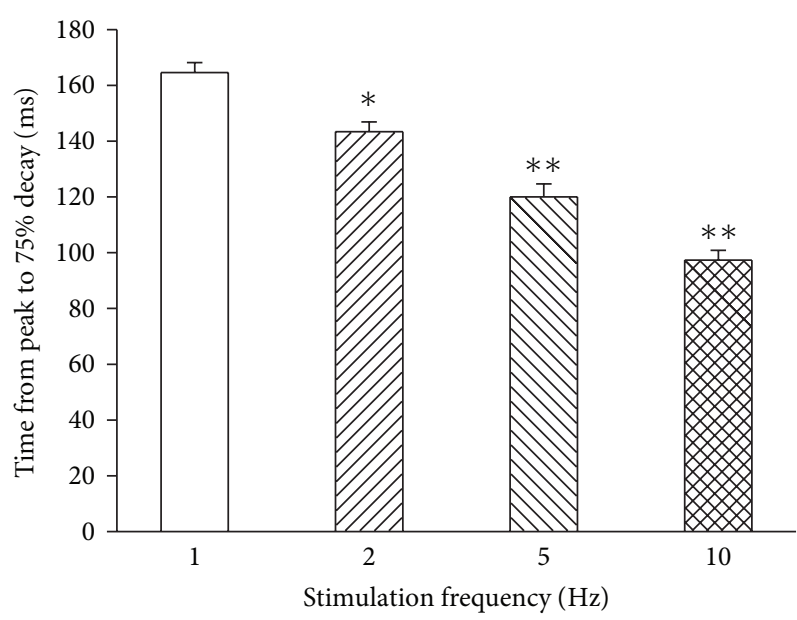

(c)

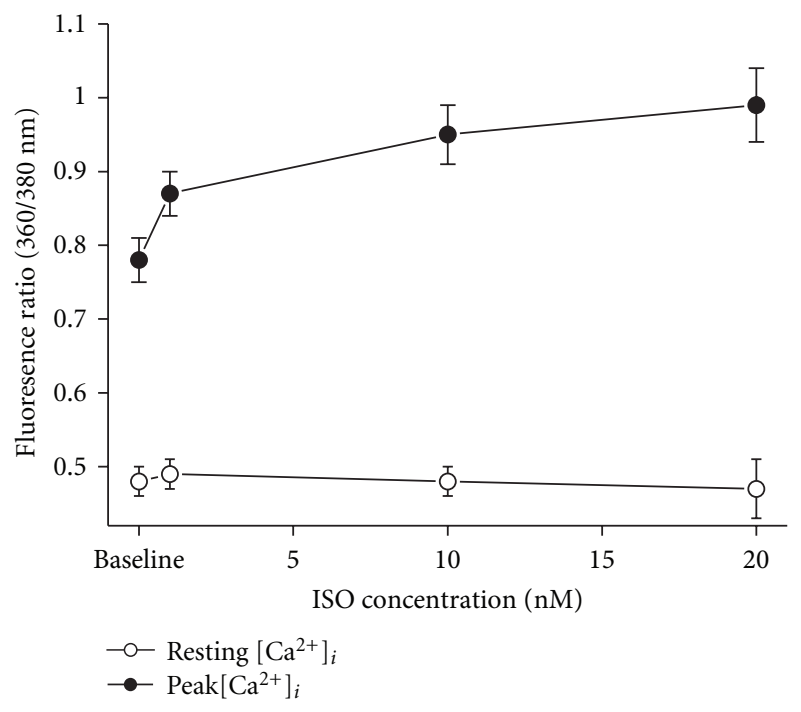

(b)

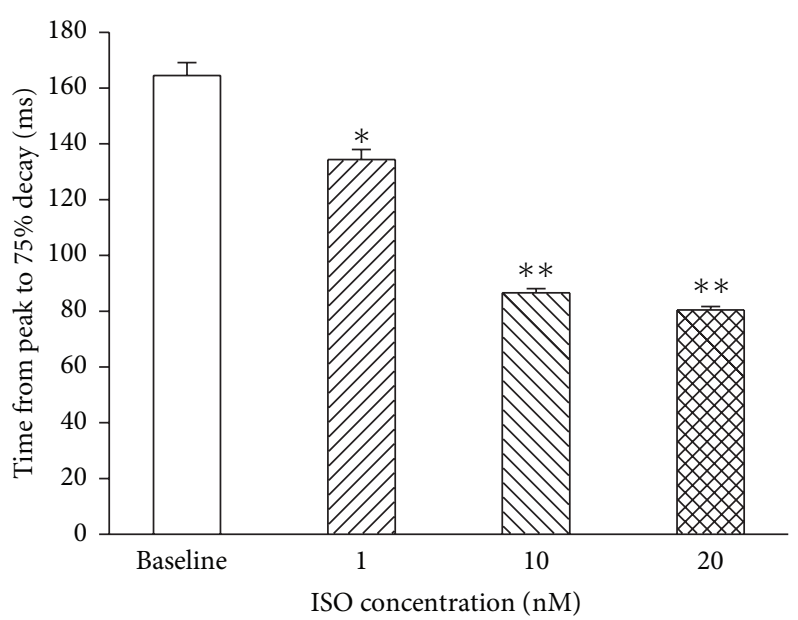

(d)

FIGURE 5: Resting and peak calcium concentrations in cardiomyocytes and time from peak to $75 \%$ decay $\left(\mathrm{TD}_{75}\right)$ under different frequencies of stimulations with and without ISO treatment. Data are mean \pm SEM, $n=$ at least 30 myocytes from six hearts for each concentration in each group. At least 5 myocytes were from each heart. ${ }^{*} P<0.05$ or $* * P<0.01$ versus baseline.

indicate that phosphorylation of cTnI by PKA may be particularly important in modulating diastolic function of hearts. Fentzke et al. constructed transgenic mice (TG-ssTnI); the myocardium expressed slow skeletal TnI (ssTnI) which lacks $\mathrm{N}$-terminal extension containing two PKA phosphorylation sites. They reported that in situ relaxation of left ventricular pressure was significantly slowed in TG-ssTnI hearts compared with the wild-type mice [22]. Kentish et al. also studied transgenic mice with ssTnI replacing cTnI and used flash photolysis of diazo-2 to rapidly remove $\mathrm{Ca}^{2+}$ from skinned cardiac muscle fibers. Myocardium expressing ssTnI had no acceleration of relaxation in $\beta$-adrenergic stimulation, suggesting that $\mathrm{cTnI}$ phosphorylation played an important role in intrinsic relaxation [23]. Li et al. reported the first quantitative estimates of the relative contributions of PLB and TnI phosphorylation to the ISO induced acceleration of relaxation in mouse hearts. The TnI phosphorylation may contribute up to $14-18 \%$ of the acceleration of relaxation in response to ISO during isometric contractions in mouse myocardium [20]. Wolska et al. observed four lines of mice expressed PLB/cTnI, PLB/ssTnI, PLBKO (PLB knockout)/cTnI, and PLBKO/ssTnI in the heart, respectively. They demonstrated that expression of ssTnI in hearts of PLB knock-out mice altered the relaxant effect of $\beta$-adrenergic stimulation [24]. The underlying mechanisms of phosphorylation of cTnI by PKA regulating relaxation of hearts may be involved in myofibril $\mathrm{Ca}^{2+}$ sensitivity [16], an increase in crossbridge cycling [23], and increased binding of cTnI 


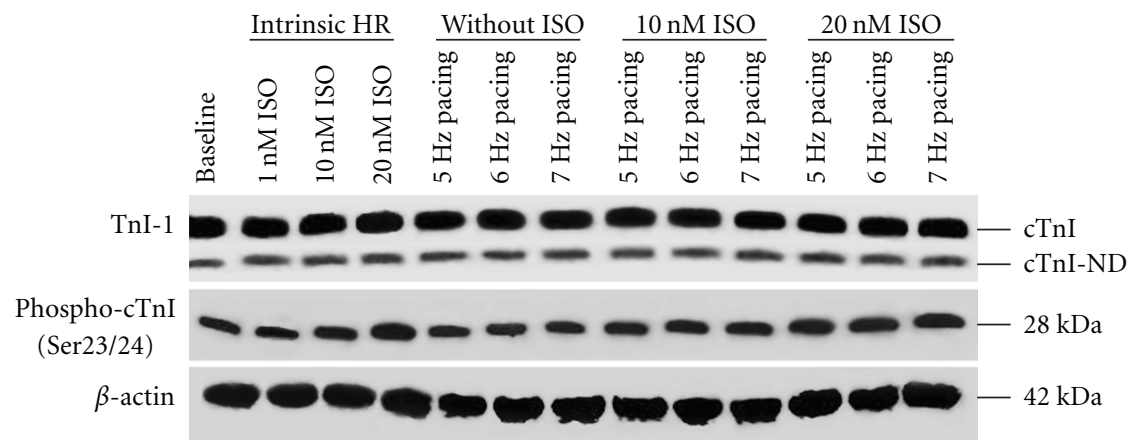

(a)

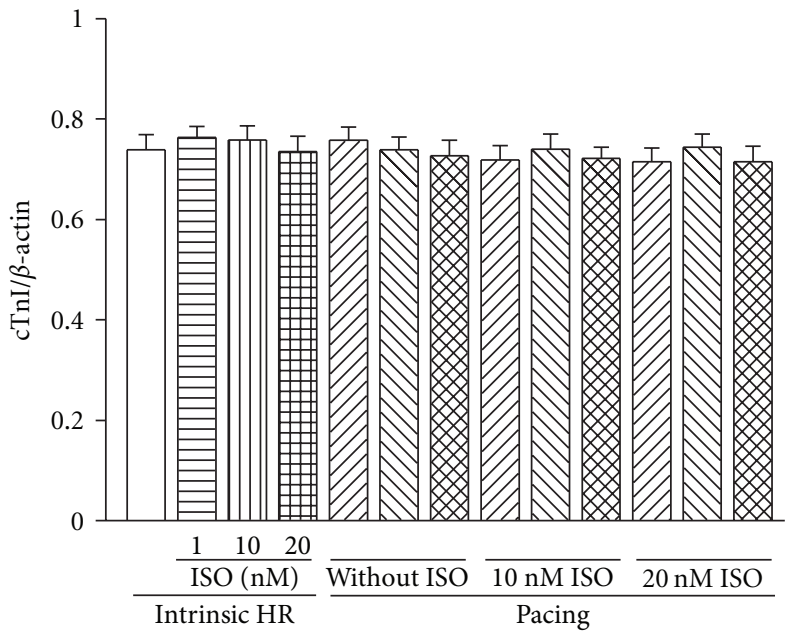

(b)

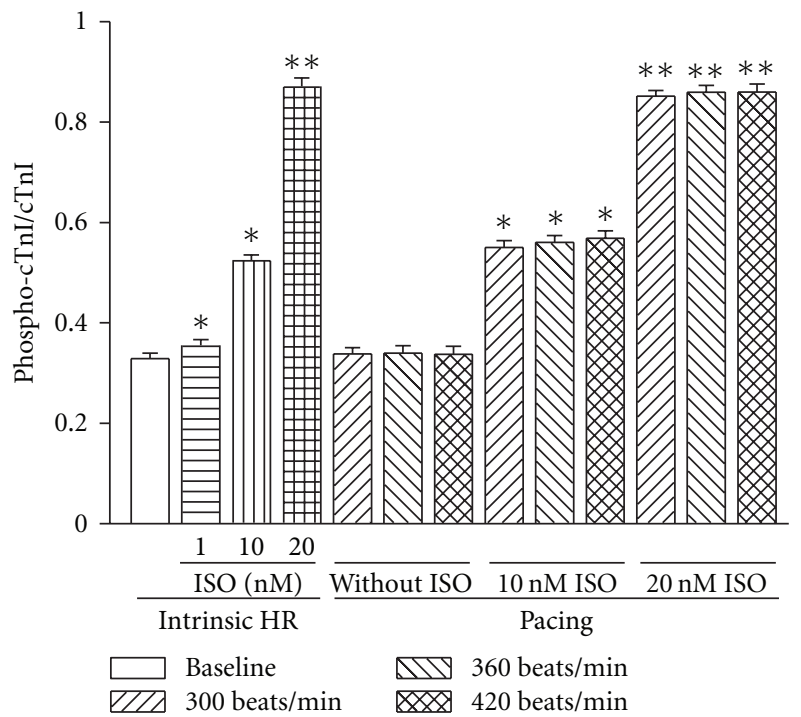

(c)

FIgure 6: Expressions of cTnI and phosphorylated cTnI at Ser23/24 in the myocardium after the pacing and ISO treatment. (a) The representative Western blots of cTnI and phosphorylated cTnI at Ser23/24. (b) Expression of total cTnI which is the sum of cTnI and $\mathrm{NH}_{2}$-terminal deleted fragment of cTnI (cTnI-ND). (c) The phosphorylation of cTnI. Data are mean \pm SEM, $n=6$ hearts in each group. ${ }^{*} P<0.05$ or ${ }^{* *} P<0.01$ versus baseline.

to the thin filament. The phosphorylation of cTnI reduces $\mathrm{Ca}^{2+}$ sensitivity of myofibrils which accelerates the rate of $\mathrm{Ca}^{2+}$ dissociation from cardiac troponin C. Taken together, phosphorylation of PLB and CTnI induces a synergistic effect to accelerate relaxation in ISO administration. Therefore, IDAR is stronger than FDAR. The less extent of FDAR elevates LVEDP which induces an insufficient diastolic filling at higher heart rate and further may reduce cardiac output.

4.2. The Phosphorylation of cTnI Causes a Synergistic Effect between PLB and cTnI and Further Enhances the Relaxation in Increasing Frequency of Stimulation. Although PKA phosphorylates many sites in the cardiomyocyte, only phosphorylated PLB and cTnI are directly related to the acceleration of relaxation in ISO stimulation. Due to the synergistic effect of PLB and cTnI, the degree of relaxation in IDAR is higher than that in FDAR. In this study, the degrees of relaxation in increasing rate of pacing showed the additional increases during ISO perfusion. Varian and Janssen reported that FDAR involved decreased myofilament calcium sensitivity
[25]. Phosphorylation of cTnI can reduce myofilament calcium sensitivity. It suggests that phosphorylation of cTnI would be helpful to FDAR. Takimoto et al. generated transgenic mice that overexpress $\mathrm{CTnI}$ in which the serine residues normally targeted by PKA were mutated to aspartic acid to mimic constitutive phosphorylation $\left(\mathrm{cTnI}-\mathrm{P}_{22} \mathrm{P}_{23}\right)$. The cTnI- $\mathrm{P}_{22} \mathrm{P}_{23}$ hearts showed a frequency-dependent enhancement of systolic and diastolic in vivo and afterload sensitivity of relaxation [26]. Using similar transgenic mice, Sakthivel et al. also demonstrated that the cTnI- $\mathrm{P}_{22} \mathrm{P}_{23}$ hearts showed augmented relaxation and contraction at higher heart rates compared with the nontransgenic controls [16].

The $10 \mathrm{nM}$ of ISO induced about $50 \%$ phosphorylation of cTnI, $45 \%$ phosphorylation of PLB at Ser-16 site, and $14 \%$ phosphorylation of PLB at Thr-17 site in the present study. These results are in agreement with the experiments of Kuschel and colleagues [27]. The phosphorylation of cTnI by ISO stimulation showed an enhancement in the extent of FDAR compared with that in the absence of ISO. While $90 \%$ of the cTnI was phosphorylated in 


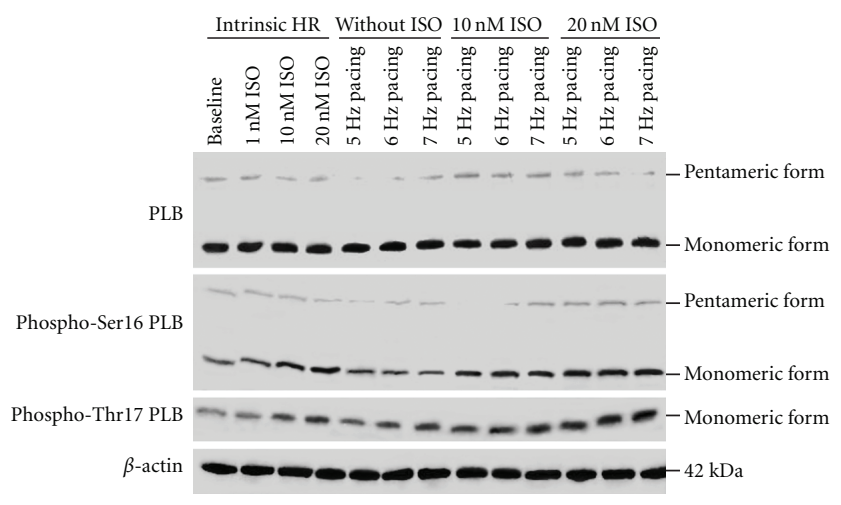

(a)

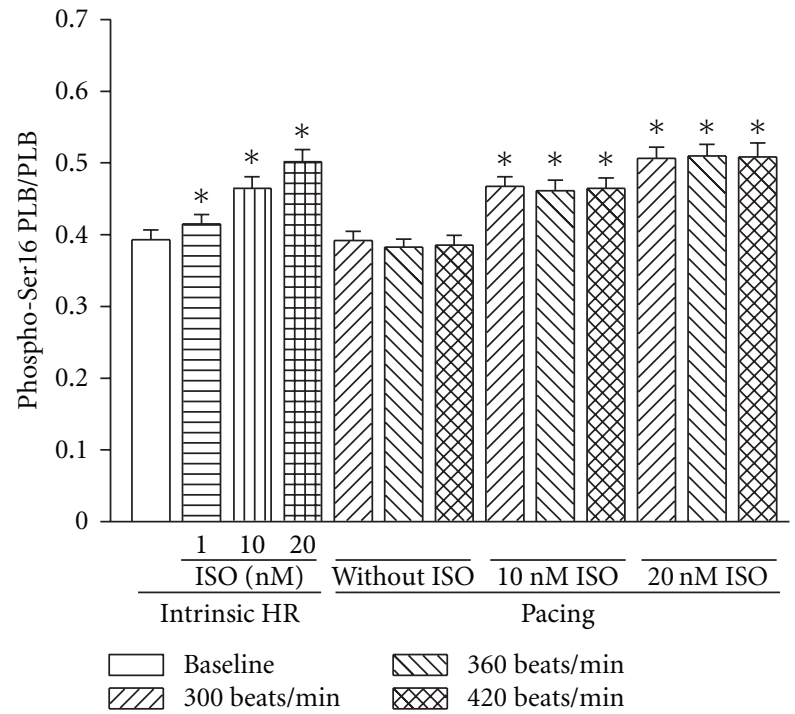

(c)

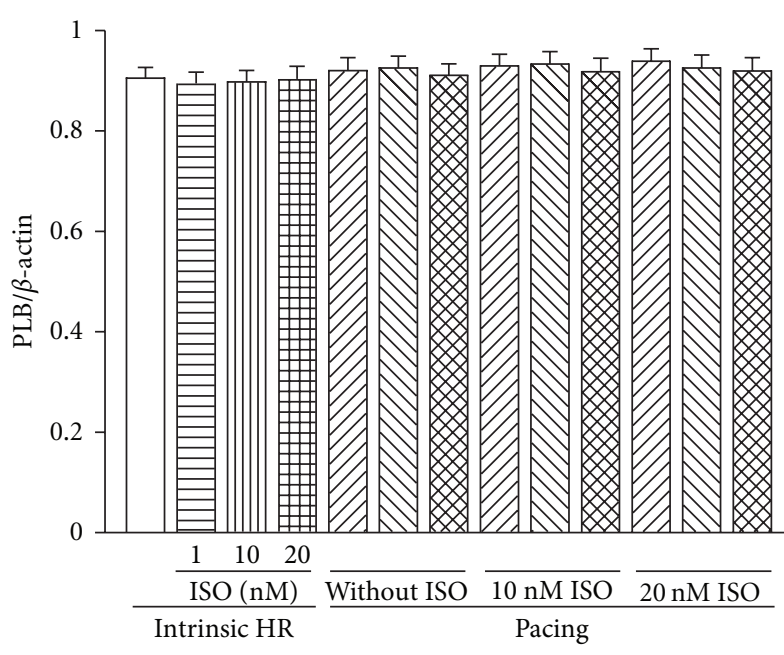

(b)

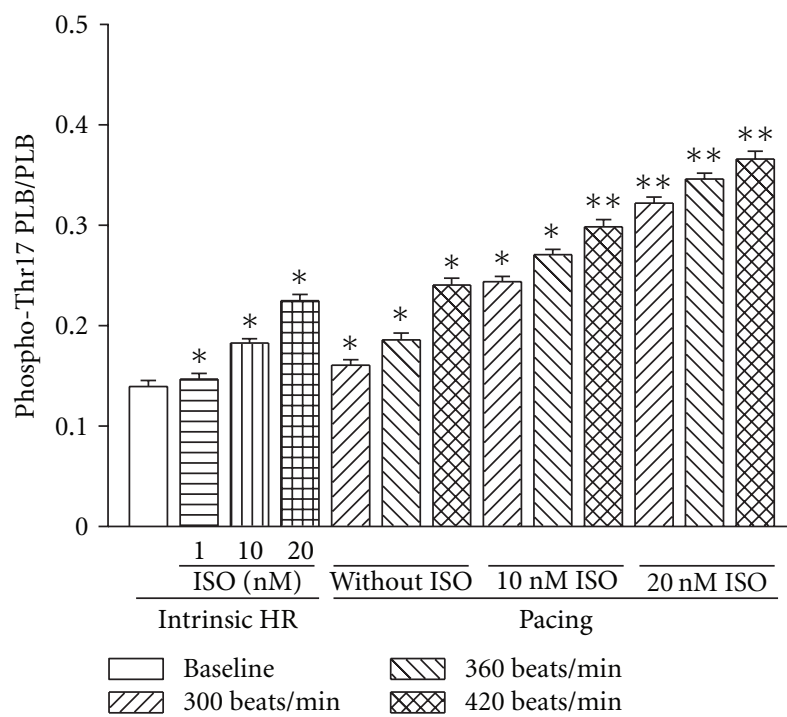

(d)

Figure 7: Expression of PLB, phosphorylated PLB at Ser16 (phospho Ser16-PLB) and at Thr17 (phospho Thr17-PLB) in the myocardium after the pacing and ISO treatment. (a) The representative Western blots of PLB, phosphorylated PLB at Ser16 and Thr17. (b) Expression of total PLB measured by pentametric plus monomeric forms. (c) Expression of phospho Ser16-PLB. (d), expression of phospho Thr17-PLB. Data are mean \pm SEM, $n=6$ hearts in each group. ${ }^{*} P<0.05$ or ${ }^{* *} P<0.01$ versus baseline.

$20 \mathrm{nM}$ of ISO treatment, the PLB Ser-16 and Thr-17 had $50 \%$ and $22 \%$ phosphorylation, respectively. The extent of FDAR in the presence of $20 \mathrm{nM}$ ISO further increased. The additional enhancement in phosphorylation of PLB Thr17 may form a synergistic effect with phosphorylation of cTnI by PKA with increasing stimulation frequency, and hence, the extent of FDAR increased. Finally, cardiac output increased in the presence of ISO rather than decreased in the absence of ISO.

In summary, this study provides evidence supporting an important role for cardiac TnI phosphorylation by PKA in the enhancement of diastolic function. And the synergistic effects between the PLB and cTnI are necessary in promoting relaxation, especially at higher heart rate.

\section{Conflicts of Interests}

The authors declare that there is no conflict of interests.

\section{Acknowledgments}

This work was supported by the National Natural Science Foundation of China Grant no. 31071044 in part to Z.-B. Yu. L. Zhang and Y. Yu contributed equally to this study.

\section{References}

[1] S. Huke and D. M. Bers, "Temporal dissociation of frequency-dependent acceleration of relaxation and protein phosphorylation by CaMKII," Journal of Molecular and Cellular Cardiology, vol. 42, no. 3, pp. 590-599, 2007. 
[2] P. de Koninck and H. Schulman, "Sensitivity of CaM kinase II to the frequency of $\mathrm{Ca}^{2+}$ oscillations," Science, vol. 279, no. 5348, pp. 227-230, 1998.

[3] T. Zhang, S. Miyamoto, and J. H. Brown, "Cardiomyocyte calcium and calcium/calmodulin-dependent protein kinase II: friends or foes?" Recent Progress in Hormone Research, vol. 59, pp. 141-168, 2004.

[4] D. R. Witcher, R. J. Kovacs, H. Schulman, D. C. Cefali, and L. R. Jones, "Unique phosphorylation site on the cardiac ryanodine receptor regulates calcium channel activity," Journal of Biological Chemistry, vol. 266, no. 17, pp. 11144-11152, 1991.

[5] A. Xu, C. Hawkins, and N. Narayanan, "Phosphorylation and activation of the $\mathrm{Ca}(2+)$-pumping ATPase of cardiac sarcoplasmic reticulum by $\mathrm{Ca}^{2+} /$ calmodulin-dependent protein kinase," Journal of Biological Chemistry, vol. 268, no. 12, pp. 8394-8397, 1993.

[6] H. K. Simmerman, J. H. Collins, and J. L. Theibert, "Sequence analysis of phospholamban. Identification of phosphorylation sites and two major structural domains," Journal of Biological Chemistry, vol. 261, no. 28, pp. 13333-13341, 1986.

[7] I. Dzhura, Y. Wu, R. J. Colbran, J. R. Balser, and M. E. Anderson, "Calmodulin kinase determines calcium-dependent facilitation of L-type calcium channels," Nature Cell Biology, vol. 2, no. 3, pp. 173-177, 2000.

[8] Y. Wu, J. Temple, R. Zhang et al., "Calmodulin kinase II and arrhythmias in a mouse model of cardiac hypertrophy," Circulation, vol. 106, no. 10, pp. 1288-1293, 2002.

[9] C. A. Valverde, C. Mundina-Weilenmann, M. Said et al., "Frequency-dependent acceleration of relaxation in mammalian heart: a property not relying on phospholamban and SERCA2a phosphorylation," Journal of Physiology, vol. 562, no. 3, pp. 801-813, 2005.

[10] D. Catalucci, M. V. Latronico, M. Ceci et al., "Akt increases sarcoplasmic reticulum $\mathrm{Ca}^{2+}$ cycling by direct phosphorylation of phospholamban at Thr17," Journal of Biological Chemistry, vol. 284, no. 41, pp. 28180-28187, 2009.

[11] A. A. Werdich, E. A. Lima, I. Dzhura et al., "Differential effects of phospholamban and $\mathrm{Ca}^{2+} /$ calmodulin- dependent kinase II on $\left[\mathrm{Ca}^{2+}\right]$ i transients in cardiac myocytes at physiological stimulation frequencies," American Journal of Physiology, vol. 294, no. 5, pp. H2352-H2362, 2008.

[12] S. M. MacDonnell, G. Garcia-Rivas, J. A. Scherman et al., "Adrenergic regulation of cardiac contractility does not involve phosphorylation of the cardiac ryanodine receptor at serine 2808," Circulation Research, vol. 102, no. 8, pp. E65-E72, 2008.

[13] J. E. Stelzer, J. R. Patel, J. W. Walker, and R. L. Moss, "Differential roles of cardiac myosin-binding protein $\mathrm{C}$ and cardiac troponin I in the myofibrillar force responses to protein kinase A phosphorylation," Circulation Research, vol. 101, no. 5, pp. 503-511, 2007.

[14] S. Sadayappan, J. Gulick, R. Klevitsky et al., "Cardiac myosin binding protein-C phosphorylation in a $\beta$-myosin heavy chain background," Circulation, vol. 119, no. 9, pp. 1253-1262, 2009.

[15] S. Sadayappan, H. Osinska, R. Klevitsky et al., "Cardiac myosin binding protein c phosphorylation is cardioprotective," Proceedings of the National Academy of Sciences of the United States of America, vol. 103, no. 45, pp. 16918-16923, 2006.

[16] S. Sakthivel, N. L. Finley, P. R. Rosevear et al., "In vivo and in vitro analysis of cardiac troponin I phosphorylation," Journal of Biological Chemistry, vol. 280, no. 1, pp. 703-714, 2005.

[17] J. C. Barbato, Q. Q. Huang, M. M. Hossain, M. Bond, and J. P. Jin, "Proteolytic N-terminal truncation of cardiac troponin
I enhances ventricular diastolic function," Journal of Biological Chemistry, vol. 280, no. 8, pp. 6602-6609, 2005.

[18] Y. Y. Wang, B. Jiao, W. G. Guo, H. L. Che, and Z. B. Yu, "Excessive thyroxine enhances susceptibility to apoptosis and decreases contractility of cardiomyocytes," Molecular and Cellular Endocrinology, vol. 320, no. 1-2, pp. 67-75, 2010.

[19] J. Desantiago, L. S. Maier, and D. M. Bers, "Frequency-dependent acceleration of relaxation in the heart depends on CamKII, but not phospholamban," Journal of Molecular and Cellular Cardiology, vol. 34, no. 8, pp. 975-984, 2002.

[20] L. Li, J. Desantiago, G. Chu, E. G. Kranias, and D. M. Bers, "Phosphorylation of phospholamban and troponin I in $\beta$-adrenergic-induced acceleration of cardiac relaxation," American Journal of Physiology, vol. 278, no. 3, pp. H769-H779, 2000.

[21] E. C. Johns, S. J. Simnett, I. P. Mulligan, and C. C. Ashley, "Troponin I phosphorylation does not increase the rate of relaxation following laser flash photolysis of diazo-2 in guineapig skinned trabeculae," Pflugers Archiv, vol. 433, no. 6, pp. 842-844, 1997.

[22] R. C. Fentzke, S. H. Buck, J. R. Patel et al., "Impaired cardiomyocyte relaxation and diastolic function in transgenic mice expressing slow skeletal troponin I in the heart," Journal of Physiology, vol. 517, no. 1, pp. 143-157, 1999.

[23] J. C. Kentish, D. T. McCloskey, J. Layland et al., "Phosphorylation of troponin I by protein kinase A accelerates relaxation and crossbridge cycle kinetics in mouse ventricular muscle," Circulation Research, vol. 88, no. 10, pp. 1059-1065, 2001.

[24] B. M. Wolska, G. M. Arteaga, J. R. Pena et al., "Expression of slow skeletal troponin I in hearts of phospholamban knockout mice alters the relaxant effect of $\beta$-adrenergic stimulation," Circulation Research, vol. 90, no. 8, pp. 882-888, 2002.

[25] K. D. Varian and P. M. L. Janssen, "Frequency-dependent acceleration of relaxation involves decreased myofilament calcium sensitivity," American Journal of Physiology, vol. 292, no. 5, pp. H2212-H2219, 2007.

[26] E. Takimoto, D. G. Soergel, P. M. L. Janssen, L. B. Stull, D. A. Kass, and A. M. Murphy, "Frequency- and afterload-dependent cardiac modulation in vivo by troponin I with constitutively active protein kinase a phosphorylation sites," Circulation Research, vol. 94, no. 4, pp. 496-504, 2004.

[27] M. Kuschel, P. Karczewski, P. Hempel, W. -P. Schlegel, E. -G. Krause, and S. Bartel, "Ser16 prevails over Thr17 phospholamban phosphorylation in the $\beta$ - adrenergic regulation of ardiac relaxation," American Journal of Physiology, vol. 276, no. 5, pp. H1625-H1633, 1999. 

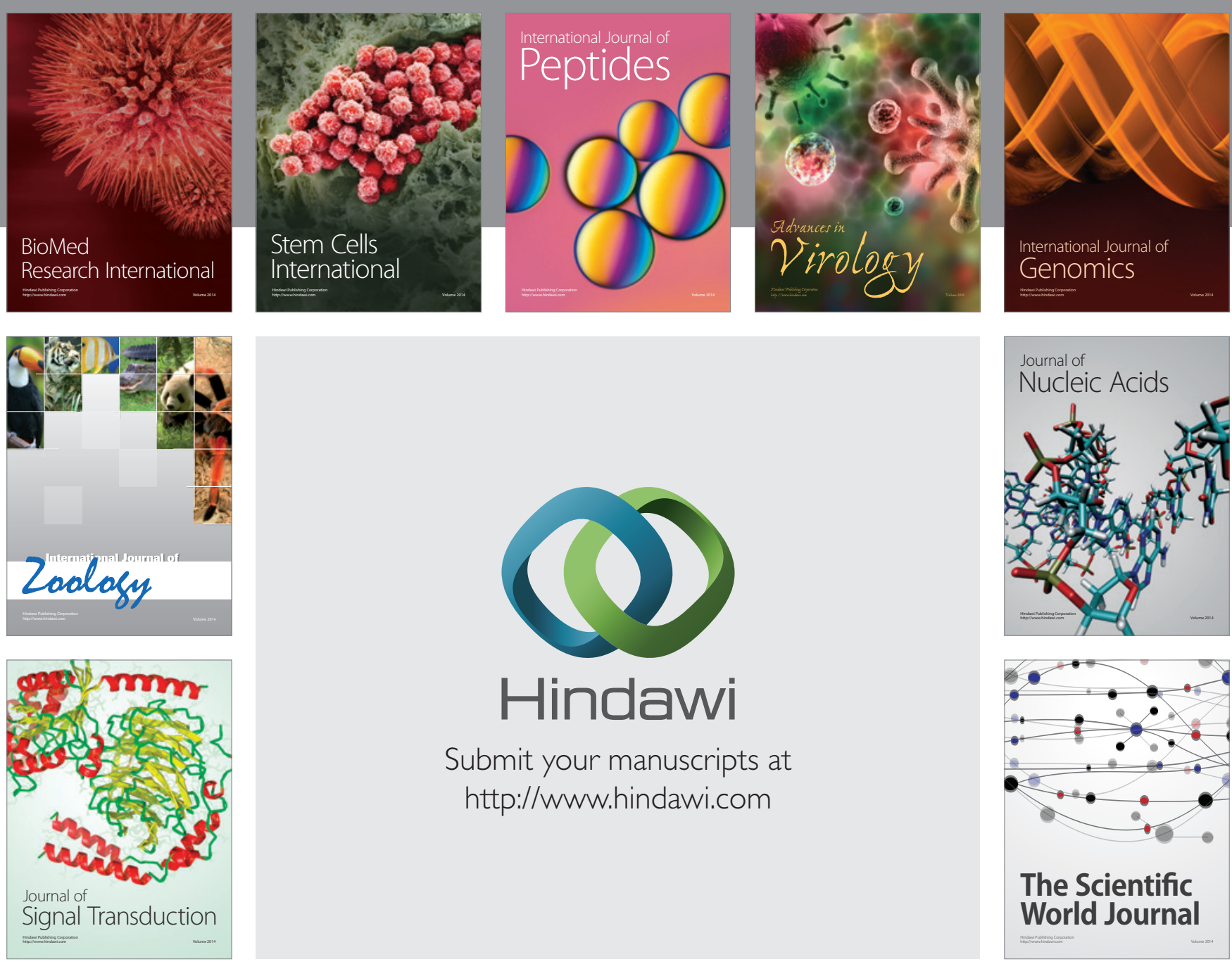

Submit your manuscripts at

http://www.hindawi.com
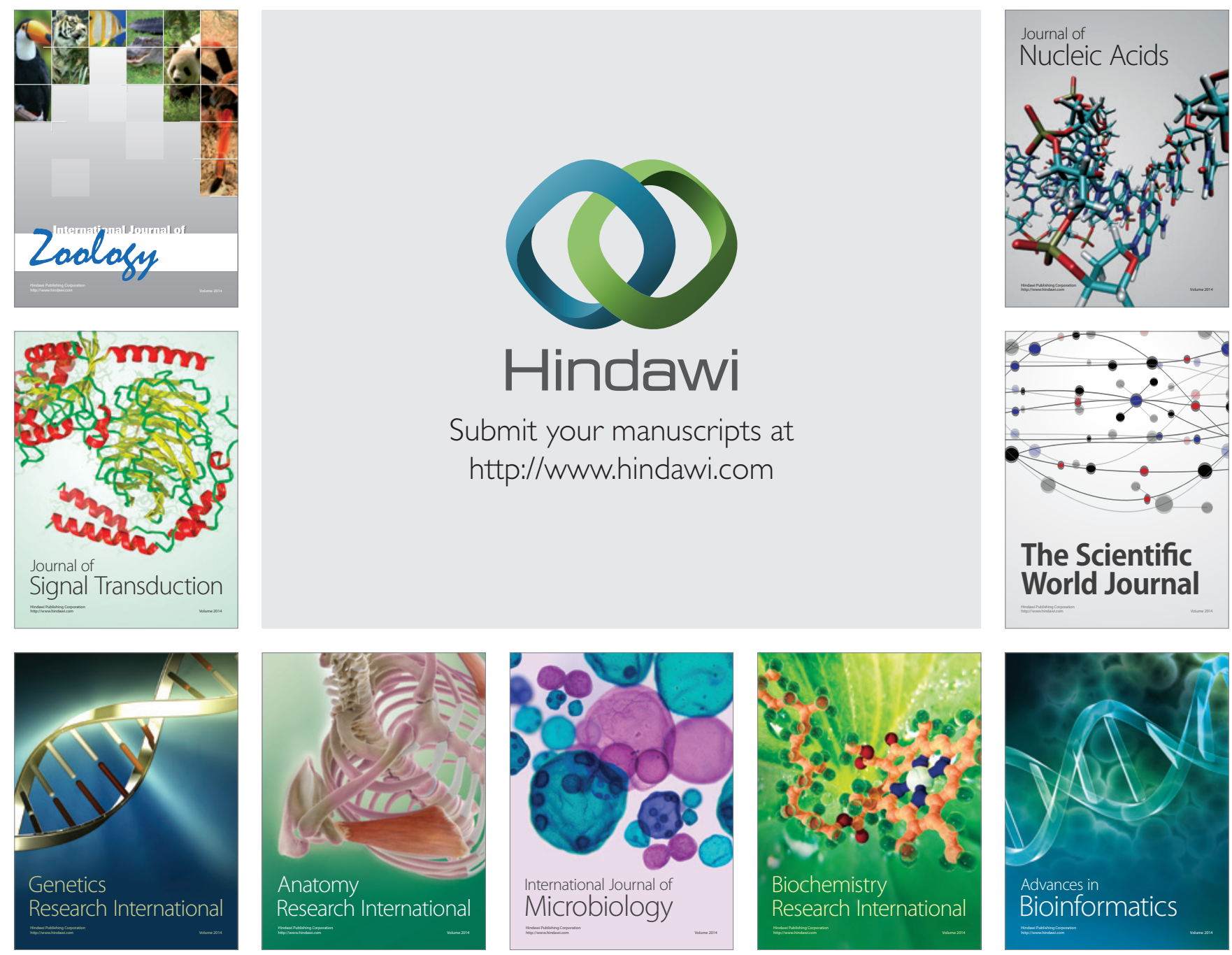

The Scientific World Journal
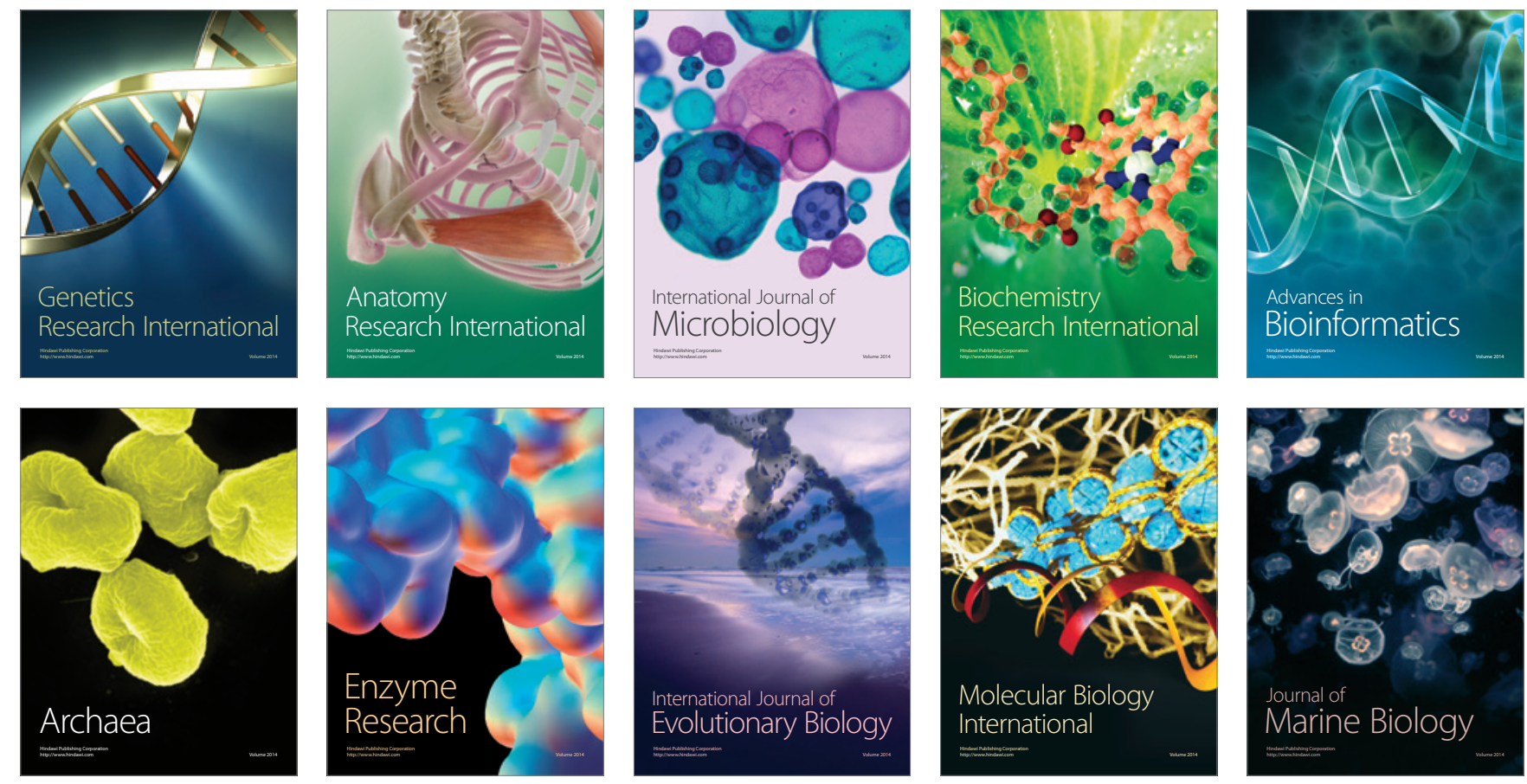